\title{
Pharmacological actions of Dieckol on the modulation of platelet functions and thrombus formation via integrin $\alpha \operatorname{Ib} \beta 3$ and cAMP signaling
}

\author{
Muhammad Irfan ${ }^{1}$, Tae-Hyung Kwon ${ }^{2}$, Hyuk-Woo Kwon ${ }^{3}$, and Man Hee Rhee ${ }^{1}$ \\ ${ }^{1}$ Kyungpook National University \\ ${ }^{2}$ Chuncheon Bio Industry Foundation \\ ${ }^{3}$ Far East University
}

June 18, 2020

\begin{abstract}
Background and purpose: Dieckol is a phlorotannin that can be found in seaweeds, particularly in Eisenia bicyclis (brown algae) and is known to have anti-oxidant, anti-inflammatory, and anti-microbial properties. It also possesses anti-thrombotic and profibrinolytic activities; however, the mechanistic aspects of anti-platelet and anti-thrombotic activity are yet to be explored. Experimental approach: We investigated the pharmacological effects of dieckol on the modulation of platelet functions using human, rat, and mice models. Inhibitory effects of dieckol on platelet aggregation were assessed using platelet-rich plasma and washed platelets, followed by measurement of dense granule secretions, fibrinogen binding to integrin $\alpha \operatorname{Ir} \beta 3$, fibronectin adhesion assay, platelet spreading on immobilized fibrinogen, and clot retraction. Cyclic nucleotide signaling events were evaluated, such as cyclic-AMP production followed by vasodilator-stimulated phosphoprotein (VASP) stimulation. The in vivo anti-thrombotic potential was evaluated in mice using an acute pulmonary thromboembolism model and tail bleeding assay. Key Results: Dieckol markedly inhibited platelet aggregation and granule secretion; furthermore, it down-regulated integrin aIIb 33 -mediated inside-out and outside-in signaling events, including platelet adhesion, spreading, and clot retraction, whereas it upregulated the cAMP-PKA-VASP pathway. Dieckol-treated mice significantly survived the thrombosis than vehicle-treated mice, without affecting hemostasis. Histological examinations of lungs revealed minimum occluded vasculature in dieckol-treated mice. Conclusion and implications: Taken together, dieckol possesses strong anti-platelet and anti-thrombotic properties and is a potential therapeutic drug candidate to treat and prevent platelet-related cardiovascular disorders.
\end{abstract}

\section{Abbreviations}

ACD, acid citrate dextrose; ADP, adenosine diphosphate; ATP, adenosine triphosphate; cAMP, 3',5'cyclic adenosine monophosphate; DMSO, dimethyl sulfoxide; PRP, platelet rich plasma; VASP, vasodilatorstimulated phosphoprotein; WP, washed platelets.

\section{What is already known}

- Impaired hemostasis is a common complication of anti-platelet drugs while dealing with thrombotic events.

- Surface integrins especially $\alpha_{\mathrm{IIb}} \beta_{3}$ play critical role in modulation of platelet functions and thormbus formation.

\section{What does this study adds}

Dieckol inhibts platelet aggregation and thrombus formation without affecting hemostasis by regulating integrin $\alpha_{\mathrm{IIb}} \beta_{3}$ and cAMP signaling. 


\section{What is the clinical significance}

Dieckol can be a potential candiate to treat and prevent platelet-related cardiovascular disorders.

\section{Introduction}

Cardiovascular disease (CVD) is considered among the leading causes of death globally. In 2013, coronary heart disease caused almost one in seven deaths, and heart failure caused one in nine deaths in the United States (Mozaffarian et al., 2016; Shafiq, Tatinati, Ang, \& Veluvolu, 2016). In Europe, CVD causes $42 \%$ of deaths in men and $52 \%$ of deaths in women (Pagidipati \& Gaziano, 2013). CVD includes a number of diseases and conditions like atherosclerosis, coronary heart disease, heart attack, hypertension, and stroke. Several risk factors are involved in the pathophysiology of CVD, but hyperactive platelets are considered main culprits as they cause hemostatic plug and stenosis of vasculature, which may also lead to ischemic stroke. Under normal physiological conditions, platelets play a vital role in maintaining hemostasis; however, aberrant or hyper-activation of platelets may cause thrombotic complications that contribute to the development of thrombosis or other platelet-related CVD (Andrews \& Berndt, 2004; J.-H. Shin, Kwon, Rhee, \& Park, 2019). Pharmacological suppression of platelets has been proven effective in the treatment and prevention of CVD; however, available treatment options are often hampered by side effects and complications. For example, aspirin is a great antiplatelet agent but may cause severe gastric ulcers or prolonged bleeding, while clopidogrel sometimes causes aplastic anemia and thrombocytopenic purpura (Barrett et al., 2008), while a significant part of population is resistant to these most commonly used anti-platelet (Ferguson, Dokainish, \& Lakkis, 2008; Wang, Bhatt, \& Topol, 2006), thus, this situation necessitates the development of safer and efficacious therapeutic regimes with no or minimum side effects. In this regard, ethno-medicinal application could be one of the best approaches to hamper CVD and CVD-related complications (Badimon, Vilahur, \& Padro, 2010). Natural herbal compounds and the Mediterranean diet reportedly possess anti-atherosclerotic and anti-thrombotic properties due to the presence of bioactive compounds affecting platelet function, thus reducing the future risk of thrombosis (Irfan, Kim, \& Rhee, 2020; J.-H. Kim, 2018; Vilahur \& Badimon, 2013).

Adenosine diphosphate (ADP) is an important in vivo agonist, affecting two types of receptors in the platelet membrane, namely $\mathrm{P}_{2} \mathrm{Y}_{1}$ and $\mathrm{P}_{2} \mathrm{Y}_{12}$. ADP causes calcium mobilization, platelet shape change, and rapid reversible platelet aggregation through $\mathrm{P}_{2} \mathrm{Y}_{1}$-mediated signaling, whereas it triggers granule secretion and stable platelet aggregation via $\mathrm{P}_{2} \mathrm{Y}_{12}$-mediated signaling pathway (Michelson, 2008), Mitogen-activated protein kinases (MAPK) and the PI3K/Akt signaling pathway have been proven to be involved in agoniststimulated platelet aggregation and thrombus formation (Kansra \& Shukla, 2000). Cyclic nucleotide signaling triggers the elevation of cyclic-AMPvia the phosphorylation of vasodilator-stimulated phosphoprotein (VASP), which is followed by the modulation of integrin $\alpha_{\mathrm{II}} \beta_{3}$-mediated inside-out and outside-in signaling, thus subsequently resulting in platelet activation and stable thrombus formation (Li, Ajdic, Eigenthaler, \& $\mathrm{Du}, 2003)$. Compounds that inhibit these signaling cascades would be very useful in treating and preventing platelet-related cardiovascular disorders.

We have previously investigated the anti-platelet and anti-thrombotic effects of Eisenia bicyclis extract and found that it contained several phlorotannins, including dieckol (Irfan, Kwon, Yun, Park, \& Rhee, 2018). Dieckol is a phlorotannin that can be found in sea weeds, particularly in Eisenia bicyclis (brown algae), Ecklonia cava, andEcklonia stolonifera (Kwon, Kim, Kim, \& Park, 2013). It is known to possess antimicrobial (Eom, Kim, \& Kim, 2012), anti-oxidant (T. Shin, Ahn, Hyun, Kim, \& Moon, 2014) and antiinflammatory (Yayeh et al., 2014) properties. A few years ago, Kim et al. (T. H. Kim, Ku, \& Bae, 2012) reported that this compound possesses anti-thrombotic and pro-fibrinolytic activities; however, mechanistic aspects of its antiplatelet effects have not been explored yet. Herein, we investigated its potential antiplatelet and anti-thrombotic effects and elucidated its underlying mechanistic aspects using human, rat, and mouse models.

\section{Materials and methods}

Extraction and isolation of dieckol from Eisenia bicyclis 
Dieckol and other phlorotannins were procured from the ethyl acetate fraction of E. bicyclis (brown algae) as our previously described detailed method (Irfan, Kwon, et al., 2018; Yayeh et al., 2014). After purification using the HPLC system, several phlorotannins were procured including dieckol, and their structure was determined by NMR. Chemical structure of dieckol is shown in Figure 1E and other phlorotannins (Figure S1) .

Chemicals and reagents

Collagen and ADP were obtained from Chrono-Log (Havertown, PA, USA). Thrombin, epinephrine, fura-2acetoxymethyl ester (fura-2/AM), and dimethyl sulfoxide (DMSO) were purchased from Sigma-Aldrich (St. Louis, MO, USA). An ATP Assay Kit was obtained from Biomedical Research Service Center (Buffalo, NY, USA). Cyclic-AMP and cyclic-GMP EIA kits were obtained from Cayman Chemicals (Ann Arbor, MI, USA), and the serotonin EIA kit was purchased from Labor Diagnostika Nord (Nordhorn, Germany). Fibrinogen Alexa Fluor 488 conjugate was obtained from Molecular Probes (Eugene, OR, USA). Fibronectin Adhesion Assay Kit was procured from Cell Biolabs, Inc. (San Diego, CA, USA). Antibodies against vasodilatorstimulated phosphoprotein (VASP, RRID:AB_2213542), phospho-VASPser157 (RRID: AB_331569), $\beta$-actin (RRID: AB_330288), phospho-ERK (phospho-p44/42, RRID: AB_331646), ERK (p44/42, RRID: AB_330744), stress-activated protein kinase (SAPK)/c-Jun N-terminal protein kinase (JNK, RRID: AB_330894), phospho-SAPK/JNK (RRID: AB_330894), Phspho-P38 ${ }^{\text {MAPK }}$ (RRID: AB_331641), P38 ${ }^{\text {MAPK }}$ (RRID: AB_330713), phospho-PI3K (phospho-p85, RRID: AB_659940), PI3K (p85, RRID: AB_329869), phospho-Akt (RRID: AB_329825), and Akt (RRID: AB_329827) were acquired from Cell Signaling Technology (Beverly, MA, USA), and PKA $\alpha \beta \gamma$ (RRID: AB_2691446) was purchased from ThermoFisher Scientific (Massachusetts, USA). Dieckol was dissolved in DMSO for further use in experiments. All chemicals were of reagent grade.

Animals

Seven-week-old male Sprague-Dawley rats (RRID: RGD_734476), weighing 250-260 g and 7 weeks old C57BL/6 mice (RRID: MGI:6199956) weighing 20-22 g were purchased from Orient Co. (Seoul, Korea). Rats and mice were acclimatized for 1 week in an air-conditioned room with a $12 \mathrm{~h} / 12 \mathrm{~h}$ light/dark cycle at a temperature and humidity of $22^{\circ} \mathrm{C} \pm 2^{\circ} \mathrm{C}$ and $55 \% \pm 10 \%$, respectively. Animal experiments were conducted following IACUC guidelines, and the protocols were approved by the Ethics Committee of College of Veterinary Medicine, Kyungpook National University, Daegu, Republic of Korea. Animal studies are reported in compliance with the ARRIVE guidelines (Kilkenny, Browne, Cuthill, Emerson, \& Altman, 2010; McGrath \& Lilley, 2015) and with the recommendations made by the British Journal of Pharmacology with all effort was taken to minimize the number of animals used and their suffering.

\section{Human Platelets Preparation}

The human platelet rich plasma (PRP) from healthy human volunteers who had provided informed consent was obtained from the Korean Red Cross Blood Center (KRBC, Daegu, Republic of Korea), and its experimental use was approved by the KRBC and the Korea National Institute for Bioethics Policy Public Institutional Review Board (PIRB) at Kyungpook National University, Daegu, Republic of Korea (PIRB171019-03). Anticoagulated PRP was centrifuged for $10 \mathrm{~min}$ at $120 \mathrm{~g}$ to remove red blood cells and centrifuged for $15 \mathrm{~min}$ at $360 \mathrm{~g}$ to obtain platelet pellets. The platelets were either resuspended in platelet-poor plasma (PPP) or washed twice with washing buffer (138-mM NaCl, 2.7-mM KCl, 12-mM NaHCO $3,0.36-\mathrm{mM}$ $\mathrm{NaH}_{2} \mathrm{PO}_{4}, 5.5-\mathrm{mM}$ glucose, and 1-mM Na 2 EDTA, $\mathrm{pH}$ 6.5). The platelets were then resuspended in suspension buffer $\left(138-\mathrm{mM} \mathrm{NaCl}, 2.7-\mathrm{mM} \mathrm{KCl}, 12-\mathrm{mM} \mathrm{NaHCO}_{3}, 0.36-\mathrm{mM} \mathrm{NaH}_{2} \mathrm{PO}_{4}, 0.49-\mathrm{mM} \mathrm{MgCl}_{2}, 5.5-\mathrm{mM}\right.$ glucose, $0.25 \%$ gelatin, $\mathrm{pH} 6.9)$ to a final concentration of $5 \times 10^{8} / \mathrm{mL}$. All of the above procedures were carried out at room temperature $\left(23^{\circ} \mathrm{C} \pm 1^{\circ} \mathrm{C}\right)$.

Preparation of washed rat platelets

Whole blood was collected from rats via heart puncture and transferred to a tube containing acid citrate dextrose (ACD) anticoagulant solution. Blood was centrifuged at $170 \mathrm{~g}$ for $7 \mathrm{~min}$ to obtain PRP. Then, PRP was further centrifuged at $350 \mathrm{~g}$ for $7 \mathrm{~min}$ to isolate washed platelets (WP). The platelet concentration was 
adjusted at $3 \times 10^{8}$ cells $/ \mathrm{mL}$ using Tyrode's buffer $\left(137-\mathrm{mM} \mathrm{NaCl}, 12-\mathrm{mM} \mathrm{NaHCO}_{3}, 5.5-\mathrm{mM}_{\mathrm{N}}\right.$ glucose, 2 -mM $\mathrm{KCl}, 1-\mathrm{mM} \mathrm{MgCl}_{2}$, and 0.3-mM $\mathrm{NaHPO}_{4}, \mathrm{pH}$ 7.4) for use in platelet aggregation assays. All preparation procedures were performed at room temperature.

Platelet aggregation assay and scanning electron microscopy analysis

Anti-platelet activity of dieckol was assessed using standard in vitro techniques. Light transmission aggregometry was performed to assess the effects of dieckol on agonist-stimulated platelet aggregation using a Chrono-Log aggregometer (Havertown, PA, USA) as previously described (Irfan, Jeong, Kwon, et al., 2018). Briefly, PRP or washed platelets were pre-incubated with various concentrations of either dieckol or vehicle for $1 \mathrm{~min}$ at $37^{\circ} \mathrm{C}$ in the presence of calcium chloride $(1-\mathrm{mM} \mathrm{CaCl} 2$ for washed platelets and $10 \mathrm{mM}$ for PRP). Then, the mixture was stimulated with an agonist (ADP, collagen or thrombin) and incubated for 5 min with continuous stirring. Vehicle (DMSO) concentration was maintained at less than $0.1 \%$.

A field emission scanning electron microscope (SU8220; Hitachi, Japan) was used to assess platelet aggregation by obtaining ultrastructure images at the Center for Scientific Instruments, Kyungpook National University, Daegu, Korea. For the platelet aggregation assay, the platelet mixture was fixed with $0.5 \%$ paraformaldehyde followed by osmium tetroxide treatment; then, it was dehydrated with ascending concentrations of ethanol, freeze dried, and then scanned as previously described (Irfan, Jeong, Kwon, et al., 2018).

$\left[\mathrm{Ca}^{2+}\right]_{\mathrm{i}}$ mobilization

The intracellular calcium ion concentration $\left(\left[\mathrm{Ca}^{2+}\right]_{i}\right)$ was evaluated with fura-2/AM as previously described (Irfan, Jeong, Saba, et al., 2018). Briefly, rat PRP was incubated with 5 - $\mu \mathrm{M}$ fura-2/AM for 60 min at. Then fura-2-loaded washed platelets were prepared and pre-incubated with dieckol for $1 \mathrm{~min}$ at in the presence of $1-\mathrm{mM} \mathrm{CaCl}_{2}$ and were subsequently stimulated with for $3 \mathrm{~min}$. Fura-2 fluorescence in the cytosol was measured using a spectrofluorometer (F-2500, Hitachi, Japan) as previously described by Schaeffer and Blaustein (Schaeffer \& Blaustein, 1989) using the following formula: $\left[\mathrm{Ca}^{2+}\right]_{i}=224 \mathrm{nM} \times\left(F-F_{\min }\right) /\left(F_{\max }\right.$ - $F$ ), wherein $224 \mathrm{nM}$ is the dissociation constant of the fura- $2-\mathrm{Ca}^{2+}$ complex, and $F_{\text {min }}$ and $F_{\text {max }}$ represent fluorescence intensity levels at very low and very high $\mathrm{Ca}^{2+}$ concentrations, respectively.

ATP release assay

Human PRP or washed rat platelets were incubated with either vehicle or various concentrations of dieckol and $\mathrm{CaCl}_{2}$ (1- $\mathrm{mM} \mathrm{CaCl}_{2}$ for washed platelets and $10-\mathrm{mM}$ for PRP) for $1 \mathrm{~min}$ at and then stimulated with . Supernatants were obtained by centrifuging the platelet mixture after the aggregation reaction terminated, and ATP secretion was measured in a luminometer (GloMax 20/20, Promega, Madison, WI, USA) using an ATP assay kit (Biomedical Research Service Center).

\section{Measurement of Serotonin Concentration}

Human PRP was pre-incubated with either vehicle or various concentrations of dieckol in the presence of $10-\mathrm{mM} \mathrm{CaCl}$ for $1 \mathrm{~min}$ at and then stimulated with ADP for 5 min under continuous stirring. After the aggregation reaction was terminated, the supernatant was obtained by centrifuging the platelet mixture, and serotonin secretion was measured with the Serotonin-EIA kit, according to manufacturer's instructions.

Measurement of cyclic-AMP and cyclic-GMP

Washed rat platelets were pre-incubated with various concentrations of dieckol, forskolin, , or vehicle in the presence of $1-\mathrm{mM} \mathrm{CaCl}_{2}$ for $1 \mathrm{~min}$ at and were then stimulated with for $5 \mathrm{~min}$ in a platelet aggregometer. The reaction was terminated, and the solution was centrifuged at $2000 \mathrm{~g}$ for $5 \mathrm{~min}$ at, and the cAMP and cGMP levels in the supernatant were determined with EIA Kits (Cayman Chemical, Ann Arbor, MI, USA) according to the manufacturer's protocol.

Flow cytometry

Fibrinogen binding was measured using flow cytometry as previously described (Irfan, Jeong, Kwon, et al., 2018). Fibrinogen binding to integrin $\alpha_{\text {IIb }} \beta_{3}$ was assessed using fluorescein isothiocyanate-labeled anti- 
fibrinogen antibodies in the presence or absence of various concentrations of dieckol. Washed rat platelets were pre-treated with dieckol and stimulated with an agonist for $5 \mathrm{~min}$. Platelets were then fixed with $0.5 \%$ paraformaldehyde for $30 \mathrm{~min}$ at $4^{\circ} \mathrm{C}$, washed three times with phosphate-buffered saline (PBS), and then suspended in PBS. Flow cytometric analysis was performed using the FACS Aria III flow cytometer(r) (Becton Dickinson Immunocytometry Systems, San Jose, CA, USA), and data were analyzed using CellQuest software (Becton Dickinson Immunocytometry Systems, San Jose, CA, USA).

Fibronectin adhesion assay

To determine the effect of dieckol on platelet adhesion, fibronectin adhesion assay was performed using Fibronectin Adhesion Assay Kit (Cell Biolabs, Inc., San Diego, CA, USA), according to the manufacturer's protocol as previously described (Irfan, Jeong, Kwon, et al., 2018). Briefly, pretreated, washed rat platelets (1 x $10^{6}$ cells $/ \mathrm{mL}$ ) were incubated in fibronectin-coated wells for $90 \mathrm{~min}$ at $37 \mathrm{degC}$ and then washed with DDW. Next, the adhered cells were stained with crystal violet for 10 min and then washed with DDW. Finally, the stained adhered cells were solubilized with extraction solution. This lysate solution was transferred to a 96-well plate, and absorbance was measured at $540 \mathrm{~nm}$ with a spectrophotometer.

Platelet spreading on immobilized fibrinogen and confocal microscopy

To examine the inhibitory effect of dieckol on platelet adhesion and spreading, platelets were allowed to adhere on immobilized fibrinogen as previously described (Sakthivel Vaiyapuri et al., 2015). Coverslips were coated with $100-\mu \mathrm{g} / \mathrm{mL}$ fibrinogen in PBS overnight at $4^{\circ} \mathrm{C}$, followed by blocking with $1 \%$ bovine serum albumin for $1 \mathrm{~h}$ at room temperature. Washed rat platelets $\left(1 \times 10^{6}\right.$ cells $\left./ \mathrm{mL}\right)$ were pretreated with vehicle, dieckol, or GR144053 [a glycoprotein IIb/IIIa (integrin $\alpha_{\mathrm{IIb}} \beta_{3}$ ) receptor antagonist] in the presence of 1-mM $\mathrm{CaCl}_{2}$ and were allowed to spread for $60 \mathrm{~min}$ at $37^{\circ} \mathrm{C}$ on fibrinogen-coated cover glass; then, they were washed and stained with SYTO-9 before analysis using a Nikon A1-R-confocal microscope.

Clot retraction in human and rat platelet-rich plasma and kinetics of clot retraction

Human PRP $(400 \mu \mathrm{L})$ was pre-incubated with vehicle, dieckol, or Y-27632 (Rock inhibitor), in the presence of $10-\mathrm{mM} \mathrm{CaCl}_{2}$ and then stimulated with thrombin $(1 \mathrm{U} / \mathrm{mL})$. Clot retraction was observed for $90 \mathrm{~min}$ at room temperature and photographed. Rat PRP $(250 \mu \mathrm{L})$ was mixed with red blood cells $(5 \mu \mathrm{L})$ and vehicle, dieckol, or Y-27632. Then, Tyrode's buffer was added to bring the volume to $1 \mathrm{~mL}$ as previously described (S. Vaiyapuri et al., 2013). Thrombin $(1 \mathrm{U} / \mathrm{mL}$ ) was added to initiate fibrin clot formation, and then, clot retraction was observed for $2 \mathrm{~h}$ at room temperature. Clot weight was measured as a marker of clot retraction. Kinetics of clot retraction were assessed as previously described (Misztal, Przesław, Rusak, \& Tomasiak, 2013). Pictures were processed with Image-J software and clot surface areas were plotted as a percentage of clot retraction.

Immunoblotting

Immunoblot analysis was performed as previously described. Washed platelets were pre-incubated with various concentrations of dieckol in the presence of $1-\mathrm{mM} \mathrm{CaCl}_{2}$ for $1 \mathrm{~min}$ at and were then stimulated with Platelet aggregation was terminated by adding lysis buffer (PRO-PREP; iNtRON Biotechnology, Seoul, Korea), and then, protein concentration was estimated using the BCS assay (PRO-MEASURE; iNtRON Biotechnology). Total platelet proteins were separated in a $10 \%$ SDS-PAGE and transferred to PVDF membranes. Membranes were blocked with 5\% skim milk, probed with respective primary and secondary antibodies, and visualized using enhanced chemiluminescence.

Survival rate study

A collagen- and epinephrine-induced acute pulmonary thromboembolism experiment was carried out in mice as previously described (Canobbio et al., 2013). Briefly, mice were randomly divided (10 mice in each group) and administered saline, dieckol (30 mg/kg i.p.), or ASA (100 mg/kg i.p.) once a day for three days. Then, $1 \mathrm{~h}$ after the final injection, the mice were challenged with $0.1 \mathrm{~mL}$ of a mixture containing collagen (500 $\mu \mathrm{g} / \mathrm{mL})$ and epinephrine $(60 \mu \mathrm{g} / \mathrm{mL})$ by smooth injection into one of the tail veins. The mortality of mice in 
each group was monitored for $10 \mathrm{~min}$, and the data are presented as the percentage of surviving animals in each treatment group. At the end of each experiment, surviving animals were euthanized with an overdose of anesthesia.

Lung histology

Two minutes after the thrombotic challenge, mice were quickly euthanized with an overdose of anesthesia, and the lungs were perfused with a fixing solution ( $10 \%$ formalin buffered with calcium carbonate). The trachea was ligated and harvested together with the lungs, and the organs were soaked in cold saline and fixed with $10 \%$ formalin for $24 \mathrm{~h}$. The lungs were then embedded in paraffin, and $5-6$ - $\mu \mathrm{m}$-thick sections were cut and stained with hematoxylin and eosin for thrombus identification. The samples were observed under a light microscope (Axio Lab A1; Carl Zeiss MicroImaging, Jena, Germany) by a histopathologist blinded to the treatment, and a minimum of 10 fields were observed in each specimen.

Mice tail bleeding assay

Male mice were randomly divided (five mice in each group) and administered with saline, dieckol (30 mg $/ \mathrm{kg}$ i.p.), or ASA (50 mg/kg i.p.) once a day for three days. After one hour of last administration, mice were anesthetized, and tail bleeding assay was performed as previously described (Endale et al., 2012). Briefly, the tail was pre-warmed for $2-3 \mathrm{~min}$ in a normal saline solution at $37^{\circ} \mathrm{C}$. The tail was precisely transected $3-5$ $\mathrm{mm}$ from the tip to induce bleeding. The distal portion of the tail was vertically immersed in normal saline solution at $37^{\circ} \mathrm{C}$. The bleeding time was recorded as the time between the start of transection to cessation of bleeding.

Statistical analysis

To assess statistical significance among observed differences, the obtained data were analyzed by one-way analysis of variance (ANOVA) followed by post-hoc Dunnett's test (SAS Institute Inc., Cary, NC, USA). The given data are presented as the mean \pm standard deviation (SD). P-values of 0.05 or less were considered statistically significant.

\section{Results}

Phlorotannin from Eisenia bicyclis inhibits platelet aggregation

Five phlorotannin compounds (6,6'-bieckol, 6,8'-bieckol, 8,8'-bieckol, dieckol, and phlorofucofuroeckol-A) were obtained from the ethyl acetate fraction of the Eisenia bicyclic extract as previously described (Irfan, Kwon, et al., 2018). We investigated whether these individual compounds inhibit agonist-induced platelet aggregation. Consequently, we found that all of them inhibited platelet aggregation, with dieckol being the most potent inhibitor of platelet aggregation (Fig. S1) . Thus, we selected dieckol for further study and elucidated its underlying mechanism of modulating platelet functions.

Dieckol inhibited agonist-stimulated platelet aggregation

Anti-platelet activity of dieckol was assessed using standard in vitro techniques. Light transmission aggregometry was performed to assess the effects of dieckol on agonist-stimulated platelet aggregation. We evaluated our compound against different ligands (collagen, ADP and thrombin) and found that in each case, dieckol inhibited agonist-induced rat platelet aggregation in a dose-dependent manner (Fig. 1A) $(p<0.001)$. Similarly, dieckol showed significant inhibitory tendency against collagen, ADP, and thrombin-stimulated human platelet aggregation in a dose-dependent manner (Fig. 1B) $(p<0.001 \& p<0.05)$. Dieckol also proved to strongly and significantly inhibit platelet aggregation in human PRP in a dose-dependent manner(Fig. 1C) $(p<0.001)$. We confirmed the anti-platelet effects of dieckol on platelet aggregation using electron microscopy. Figure 1D shows platelet shape changes in vehicle or dieckol-treated platelets. Figure 1D(a) displays resting platelets, whereas Figure 1D(b) shows fully activated platelets and fibrin meshwork in vehicle-treated platelets stimulated with ADP while increasing concentrations of dieckol inhibited shape change and platelet aggregation in a dose-dependent manner [Figure 1D(c-e)]. 
Dieckol inhibited $\left[\mathrm{Ca}^{2+}\right]_{\mathrm{i}}$ elevation, and dense granule secretion

To assess whether our compound inhibited $\left[\mathrm{Ca}^{2+}\right]_{i}$ mobilization, an intracellular calcium mobilization assay was performed. As shown inFigure 2A , dieckol markedly blocked $\left[\mathrm{Ca}^{2+}\right]_{i}$ mobilization in ADP-stimulated rat platelets in a dose-dependent manner $(p<0.001)$. Dieckol has also shown inhibitory effects on ATP secretion (a marker for dense granule secretions). Dieckol significantly hampered ATP release $(p<0.01$ and $p<0.001$ ) in rats' washed platelets (Fig. 2B ) and human PRP (Fig. 2D ) in a dose-dependent manner. Similar inhibitory effects were observed on serotonin secretion from platelets in agonist-stimulated human PRP pre-treated with various concentrations of dieckol than vehicle treated platelets (Fig. 2C ).

Dieckol attenuates fibrinogen binding and fibronectin adhesion

Our results show that various concentrations of dieckol significantly inhibited fibrinogen binding to integrin $\alpha_{\mathrm{IIb}} \beta_{3}$ in a dose-dependent manner (Fig. 3A\&B ). Also, it was observed that dieckol significantly inhibited platelet adhesion to fibronectin in a dose-dependent manner(Fig. 3C) and also attenuated platelet spreading on fibrinogen(Fig. 3D) . These results suggest that dieckol blocks integrin-mediated inside-out signaling and the early phase of outside-in signaling.

Dieckol inhibits clot retraction

A chain of signaling events started after fibrinogen bound to integrin proteins; this transduced signals into the cell and initiated platelet spreading in the early phase, whereas in later phases of thrombus formation, this triggers clot retraction, which may be termed as late-stage outside-in signaling (Tucker, Sage, \& Gibbins, 2012). Our results show that dieckol treatment significantly limited thrombin-induced clot retraction in rat PRP(Fig. 3E-G) and in human PRP (Fig. 3H) .

Dieckol attenuated MAPK and PI3K/Akt phosphorylation

To explain the underlying mechanism of the effect of dieckol on ADP-stimulated platelet activation, we investigated the modulation of the MAPK pathway. Figure 4 shows that dieckol significantly attenuated the activation of ERK and JNK but not of $\mathrm{p} 38^{\mathrm{MAPK}}$; furthermore, it also inhibited PI3K/Akt phosphorylation in agonist-induced platelets in a dose-dependent manner.

Dieckol elevates intracellular cAMP level

Platelet activation suppresses cyclic-AMP generation and cyclic nucleotide-dependent protein kinase activity, whereas the elevation of intracellular cAMP levels has been proven to inhibit platelet aggregation. Therefore, we measured the inhibitory effects of dieckol on cAMP and cGMP generation and platelet activation. Our initial screening results showed that dieckol only elevates cAMP levels and had no effect on cGMP levels (Fig. 5A) . Further, cAMP levels were found to be dose-dependently increased in the presence of increasing concentrations of dieckol (Fig. 5B ).

Dieckol enhanced VASP and PKA $\alpha \gamma$ phosphorylation

VASP is a regulator of actin dynamics in platelets and is a substrate of cyclic nucleotide (cAMP/cGMP)dependent protein kinases (i.e., PKA/PKG), and the activation of VASP can hinder platelet aggregation due to the enhanced activity of these kinases. Therefore, we investigated the effect of our compound on VASP phosphorylation. The results showed that dieckol and forskolin (i.e. , a positive regulator of adenylyl cyclase) significantly enhanced the phosphorylation of total VASP as well as VASPser157 (a site preferred by PKA). To further explore whether these effects are mediated by the cAMP-PKA-VASP ${ }^{\text {ser157 }}$ pathway, we evaluated PKA $\alpha \gamma$ phosphorylation, which was significantly augmented by various ascending concentrations of dieckol (Fig. 5C).

Dieckol protects mice from thrombosis without affecting hemostasis

Our results clearly show that dieckol considerably protected mice from thrombosis and offered a significantly better survival rate (8/10) compared with the saline-treated control group (3/10) mice. Histological analysis revealed the occluded pulmonary vessels formed by platelet aggregation in the control group, whereas dieckol 
treatment greatly reduced thrombosis events. The number of occluded vessels was also observed to be far lesser in intervention group mice than in control group mice. Bleeding time was also increased in aspirintreated mice group, whereas saline and dieckol treatment did not prolonged tail bleeding (Fig. 6) . These results suggest that dieckol protected mice from thrombosis without affecting hemostasis.

\section{Discussion}

Platelets play an important role in sealing injured blood vessels by forming a hemostatic plug and maintain hemostasis; however, pathophysiological activation may lead to serious thrombotic complications, including development of atherothrombosis and thromboembolism. Cardiovascular disorders are among leading causes of morbidity and mortality in modern societies, particularly in Western countries, due to high rates of heart attack and stroke. Although several pharmacological drugs are available to treat platelet-induced CVD, they are associated with adverse effects and often lack efficacy in some patients (S. Vaiyapuri et al., 2013). It has been reported that natural therapeutic regimes and nutraceuticals are effective in reducing the CVD risk, and these approaches have gained clinical interest in treating CVD because of their proven pharmacological effects (Badimon et al., 2010; Vilahur \& Badimon, 2013). Dieckol is a natural phlorotannin found in marine algae, particularly inEisenia bicyclis, which is a popular sea food in Korea, Japan, and China and reportedly possesses several pharmacological properties. In the present study, we evaluated its anti-platelet and antithrombotic potential. First, we analyzed various phlorotannins contained in theEisenia bicyclis extract and found dieckol to be most potent among them.

Collagen, thrombin and ADP induce platelet aggregation by triggering granule secretion, platelet activation, and aggregation. These agonists or adhesive proteins cause platelet activation by triggering various intracellular signaling pathways which can be divided into the following: (i) early receptor signaling, (ii) merging common signal transduction and amplification, (iii) inside-out signaling (mainly involves conformational change in integrin $\alpha_{\mathrm{II}} \beta_{3}$ structure and causes fibrinogen binding and platelet adhesion), and (iv) outside-in signaling, which enhances platelet aggregation and triggers clot retraction (Bye et al., 2015; Estevez \& Du, 2017). Our results showed that dieckol significantly inhibited platelet aggregation in human PRP and washed platelets and also inhibited rat platelet aggregation induced by various agonists.

Platelet contains dense $(\delta)$ and alpha $(\alpha)$ granules. $\delta$ granules include ADP, ATP, Ca2+, and serotonin, whereas $\alpha$ granules contain fibronectin, fibrinogen, $v W F$, and P-selectin. Several signaling events contribute to granule secretion, such as: (i) Src family kinase signaling (considered as early signaling in platelet activation), (ii) intracellular calcium ion mobilization, (iii) cyclic nucleotide signaling (e.g., cAMP-VASP-PKA pathway); inside-out and outside-in signaling, (iv) PKC activation, (v) MAPK and PI3K/Akt activation, and (vi) RhoA pathway activation. All of the above mentioned signaling events contribute to granule secretion, which causes enhanced platelet activation and shape changes (Estevez \& Du, 2017). $\left[\mathrm{Ca}^{2+}\right]_{i}$ mobilization plays a key role in the modulation of platelet function that triggers other signaling cascades involved in coagulation, primes platelet aggregation, and thrombus formation (Smith, Negrelli, Manek, Hawes, \& Viera, 2015). In our results, dieckol markedly reduced $\left[\mathrm{Ca}^{2+}\right]_{i}$ mobilization and ATP and serotonin secretion, which suggest its potential anti-platelet mechanism.

Platelets express various integrin proteins on their surface, including $\alpha_{\mathrm{IIb}} \beta_{3}$; conformational changes in this protein during platelet activation causes fibrinogen to bind to it, which is considered a marker for inside-out signaling (Calderwood, 2004). Fibronectin, which is another adhesive ligand, binds to integrins and enhances platelet aggregation by forming cohesive aggregates that lead to stable thrombus formation in vasculature by recruiting signaling proteins in the vicinity of integrin cytoplasmic tails (Varga-Szabo, Pleines, \& Nieswandt, 2008). Dieckol attenuated fibrinogen binding, fibronectin adhesion, and platelet spreading. Further, small GTP proteins belong to the Rho family and control the cell shape, motility, and proliferation; further, Rho kinases (ROCKs) were found to activate Rho-A followed by cytoskeletal changes via myosin light chain reaction (MLC) phosphorylation. In addition, a role of Rho kinase in mediating clot retraction has been previously described using a Rho kinase inhibitor (Y-27632) (Liao, Seto, \& Noma, 2007). Dieckol inhibited clot retraction in both rat and human PRP. These results suggest that dieckol potentially inhibits platelet aggregation by inhibiting granule secretion and modulating integrin $\alpha_{\mathrm{IIb}} \beta_{3}$ signaling. Figure 7 summarizes 
the potential anti-platelet effects of dieckol on platelet intracellular signaling.

Adam et al. (Adam, Kauskot, Rosa, \& Bryckaert, 2008) have established that MAPK molecules (ERK, JNK and $\mathrm{p} 38^{\mathrm{MAPK}}$ ) activated in platelets by various agonists contribute to platelet aggregation. PI3K/Akt has been reported to lie downstream of SFK and is known to recruit and activate PLC $\gamma 2$, which consequently liberates IP3 and DAG, thus leading to calcium mobilization, granule secretion, and platelet aggregation (Senis, Mazharian, \& Mori, 2014). Our results have shown that dieckol inhibited platelet activation by inhibiting the phosphorylation of ERK, JNK, and PI3K/Akt (Fig. 7) .

Elevated levels of cAMP have been known to inhibit platelet activation via increased phosphorylation of VASP ${ }^{\text {ser157}}$; moreover, VASP is a major substrate for cAMP-dependent PKA. VASP stimulation is also known to inhibit integrin $\alpha_{\mathrm{IIb}} \beta_{3}$ signaling (Coles et al., 2002). Our results clearly state that dieckol upregulated the cAMP-PKA-VASP pathway in a dose-dependent manner, thus indicating its potential inhibitory mechanism on platelet activation (Fig. 7) .

Strong coagulation factor (e.g. , thrombin, ADP, or collagen combined with epinephrine)-induced thrombosis models via systematic administration have been widely used to evaluate anti-thrombotic drugs (Miao, Liu, \& Wang, 2010). Studies have shown that the intravenous administration of collagen and epinephrine into mice veins induced platelet aggregation and very lethal pulmonary thromboembolism in a short period of time (Huang, Wang, Luo, Xie, \& Shi, 2007). Konstantinides et al.(Konstantinides, Schafer, Neels, Dellas, \& Loskutoff, 2004) reported that the histological analysis of collagen-epinephrine-induced pulmonary thromboembolism in the lungs showed occluded vasculature rich in platelet thrombi. Anti-thrombotic effect of dieckol was evaluated by challenging mice with collagen-epinephrine intravenous injection, an established model to study defects of platelet functions and thrombosis (Miao et al., 2010). Pulmonary thromboembolism was observed in the majority of controlled mice, whereas dieckol- and ASA-treated mice significantly survived the thrombosis. Moreover, dieckol did not increase the bleeding time, indicating that it modulated platelet function without affecting hemostasis.

\section{Conclusion}

Dieckol significantly inhibited agonist-induced human and rat platelet aggregation, granule secretion, and integrin $\alpha_{\mathrm{II}} \beta_{3}$-mediated inside-out and outside-in signaling; furthermore, it down-regulated MAPK and PI3K/Akt pathways but upregulated the cAMP-PKA-VASP pathway. Moreover, it protected mice from thrombosis without affecting hemostasis. Taken together, these results indicate the clinical significance and potential of dieckol as an anti-platelet and anti-thrombotic agent for treatment and prevention of plateletrelated cardiovascular disorders.

\section{Author Contributions}

MI and MHR designed and conceptualized the study. MI performed experiments, analyzed data and wrote manuscript. H-WK and T-HK performed partial experiments and analyzed data. MHR and T-HK supervised the research work. All authors read and approved the final manuscript.

\section{Declaration of Transparency and Scientific Rigour}

This Declaration acknowledges that this paper adheres to the principles for transparent reporting and scientific rigour of preclinical research as stated in the BJP guidelines for Design \& Analysis and Animal Experimentation, and as recommended by funding agencies, publishers and other organizations engaged with supporting research.

\section{References}

Adam, F., Kauskot, A., Rosa, J. P., \& Bryckaert, M. (2008). Mitogen-activated protein kinases in hemostasis and thrombosis. Journal of Thrombosis and Haemostasis, 6 (12), 2007-2016. doi:10.1111/j.15387836.2008.03169.x 
Andrews, R. K., \& Berndt, M. C. (2004). Platelet physiology and thrombosis. Thrombosis Research, 114 (5-6), 447-453. doi:10.1016/j.thromres.2004.07.020

Badimon, L., Vilahur, G., \& Padro, T. (2010). Nutraceuticals and atherosclerosis: human trials.Cardiovascular Therapeutics, 28 (4), 202-215. doi:10.1111/j.1755-5922.2010.00189.x

Barrett, N. E., Holbrook, L., Jones, S., Kaiser, W. J., Moraes, L. A., Rana, R., . . . Gibbins, J. M. (2008). Future innovations in anti-platelet therapies. British Journal of Pharmacology, 154 (5), 918-939. doi:10.1038/bjp.2008.151

Bye, A. P., Unsworth, A. J., Vaiyapuri, S., Stainer, A. R., Fry, M. J., \& Gibbins, J. M. (2015). Ibrutinib inhibits platelet integrin $\alpha \operatorname{IIb} \beta 3$ outside-in signaling and thrombus stability but not adhesion to collagen. Arteriosclerosis Thrombosis and Vascular Biology, 35 (11), 2326-2335.

Calderwood, D. A. (2004). Integrin activation. Journal of Cell Science, 117 (Pt 5), 657-666. doi:10.1242/jcs.01014

Canobbio, I., Cipolla, L., Consonni, A., Momi, S., Guidetti, G., Oliviero, B., . . . Torti, M. (2013). Impaired thrombin-induced platelet activation and thrombus formation in mice lacking the $\mathrm{Ca}(2+)$-dependent tyrosine kinase Pyk2. Blood, 121 (4), 648-657. doi:10.1182/blood-2012-06-438762

Coles, B., Bloodsworth, A., Eiserich, J. P., Coffey, M. J., McLoughlin, R. M., Giddings, J. C., . . . O'Donnell, V. B. (2002). Nitrolinoleate inhibits platelet activation by attenuating calcium mobilization and inducing phosphorylation of vasodilator-stimulated phosphoprotein through elevation of cAMP. Journal of Biological Chemistry, 277 (8), 5832-5840.

Endale, M., Lee, W., Kamruzzaman, S., Kim, S., Park, J., Park, M., . . . Rhee, M. (2012). Ginsenoside-Rp1 inhibits platelet activation and thrombus formation via impaired glycoprotein VI signalling pathway, tyrosine phosphorylation and MAPK activation. British Journal of Pharmacology, 167 (1), 109-127.

Eom, S.-H., Kim, Y.-M., \& Kim, S.-K. (2012). Antimicrobial effect of phlorotannins from marine brown algae.Food and Chemical Toxicology, 50 (9), 3251-3255.

Estevez, B., \& Du, X. (2017). New Concepts and Mechanisms of Platelet Activation Signaling.Physiology (Bethesda, Md.), 32 (2), 162-177. doi:10.1152/physiol.00020.2016

Ferguson, A. D., Dokainish, H., \& Lakkis, N. (2008). Aspirin and clopidogrel response variability: review of the published literature. Texas Heart Institute Journal, 35 (3), 313.

Huang, J., Wang, S., Luo, X., Xie, Y., \& Shi, X. (2007). Cinnamaldehyde reduction of platelet aggregation and thrombosis in rodents. Thrombosis Research, 119 (3), 337-342.

Irfan, M., Jeong, D., Kwon, H.-W., Shin, J.-H., Park, S.-J., Kwak, D., . . . Rhee, M. H. (2018). GinsenosideRp3 inhibits platelet activation and thrombus formation by regulating MAPK and cyclic nucleotide signaling. Vascular pharmacology, 109 , 45-55.

Irfan, M., Jeong, D., Saba, E., Kwon, H.-W., Shin, J.-H., Jeon, B.-R., . . . Nah, S.-Y. (2018). Gintonin modulates platelet function and inhibits thrombus formation via impaired glycoprotein VI signaling. Platelets, $30(5), 589-598$.

Irfan, M., Kim, M., \& Rhee, M. H. (2020). Anti-platelet role of Korean ginseng and ginsenosides in cardiovascular diseases. Journal of Ginseng Research, 44 (1), 24-32.

Irfan, M., Kwon, T.-H., Yun, B.-S., Park, N.-H., \& Rhee, M. H. (2018). Eisenia bicyclis (brown alga) modulates platelet function and inhibits thrombus formation via impaired P2Y12 receptor signaling pathway. Phytomedicine, $40,79-87$.

Kansra, S. V., \& Shukla, S. D. (2000). Reverse relationship between mitogen activated protein kinase and human platelet aggregation. Clinical and Experimental Hypertension, 22 (2), 145-154. 
Kilkenny, C., Browne, W., Cuthill, I. C., Emerson, M., \& Altman, D. G. (2010). Animal research: reporting in vivo experiments: the ARRIVE guidelines. British Journal of Pharmacology, 160 (7), 1577-1579.

Kim, J.-H. (2018). Pharmacological and medical applications of Panax ginseng and ginsenosides: a review for use in cardiovascular diseases. Journal of Ginseng Research, 42 (3), 264-269.

Kim, T. H., Ku, S. K., \& Bae, J. S. (2012). Antithrombotic and profibrinolytic activities of eckol and dieckol. Journal of cellular biochemistry, 113 (9), 2877-2883.

Konstantinides, S., Schafer, K., Neels, J. G., Dellas, C., \& Loskutoff, D. J. (2004). Inhibition of endogenous leptin protects mice from arterial and venous thrombosis. Arteriosclerosis Thrombosis and Vascular Biology, 24 (11), 2196-2201.

Kwon, T. H., Kim, T. W., Kim, C. G., \& Park, N. H. (2013). Antioxidant activity of various solvent fractions from edible brown alga, Eisenia bicyclis and its active compounds. Journal of food science, 78 (5), C679-C684.

Li, Z., Ajdic, J., Eigenthaler, M., \& Du, X. (2003). A predominant role for cAMP-dependent protein kinase in the cGMP-induced phosphorylation of vasodilator-stimulated phosphoprotein and platelet inhibition in humans. Blood, 101 (11), 4423-4429.

Liao, J. K., Seto, M., \& Noma, K. (2007). Rho kinase (ROCK) inhibitors. Journal of Cardiovascular Pharmacology, 50 (1), 17.

McGrath, J. C., \& Lilley, E. (2015). Implementing guidelines on reporting research using animals (ARRIVE etc.): new requirements for publication in BJP. British Journal of Pharmacology, 172 (13), 3189-3193.

Miao, R., Liu, J., \& Wang, J. (2010). Overview of mouse pulmonary embolism models. Drug Discovery Today: Disease Models, 7 (3-4), 77-82.

Michelson, A. D. (2008). P2Y12 Antagonism. Arteriosclerosis, Thrombosis, and Vascular Biology, 28 (3), s33-s38.

Misztal, T., Przesław, K., Rusak, T., \& Tomasiak, M. (2013). Peroxynitrite-altered platelet mitochondriaA new link between inflammation and hemostasis. Thrombosis Research, 131 (1), e17-e25.

Mozaffarian, D., Benjamin, E. J., Go, A. S., Arnett, D. K., Blaha, M. J., Cushman, M., . . . Fullerton, H. J. (2016). Executive summary: heart disease and stroke statistics - 2016 update: a report from the American Heart Association. Circulation, 133 (4), 447-454.

Pagidipati, N. J., \& Gaziano, T. A. (2013). Estimating deaths from cardiovascular disease: a review of global methodologies of mortality measurement. Circulation, 127 (6), 749-756.

Schaeffer, J., \& Blaustein, M. P. (1989). Platelet free calcium concentrations measured with fura-2 are influenced by the transmembrane sodium gradient. Cell Calcium, 10 (2), 101-113.

Senis, Y. A., Mazharian, A., \& Mori, J. (2014). Src family kinases: at the forefront of platelet activation.Blood, 124 (13), 2013-2024. doi:10.1182/blood-2014-01-453134

Shafiq, G., Tatinati, S., Ang, W. T., \& Veluvolu, K. C. (2016). Automatic identification of systolic time intervals in seismocardiogram. Scientific Reports, 6 , 37524.

Shin, J.-H., Kwon, H.-W., Rhee, M. H., \& Park, H.-J. (2019). Inhibitory effects of thromboxane A2 generation by ginsenoside Ro due to attenuation of cytosolic phospholipase A2 phosphorylation and arachidonic acid release. Journal of Ginseng Research, 43 (2), 236-241. doi:https://doi.org/10.1016/j.jgr.2017.12.007.

Shin, T., Ahn, M., Hyun, J. W., Kim, S. H., \& Moon, C. (2014). Antioxidant marine algae phlorotannins and radioprotection: a review of experimental evidence. Acta histochemica, 116 (5), 669-674. 
Smith, J. N., Negrelli, J. M., Manek, M. B., Hawes, E. M., \& Viera, A. J. (2015). Diagnosis and management of acute coronary syndrome: an evidence-based update. Journal of the American Board of Family Medicine, 28 (2), 283-293. doi:10.3122/jabfm.2015.02.140189

Tucker, K. L., Sage, T., \& Gibbins, J. M. (2012). Clot retraction. Methods in Molecular Biology, 788 , 101-107. doi:10.1007/978-1-61779-307-3_8

Vaiyapuri, S., Ali, M. S., Moraes, L. A., Sage, T., Lewis, K. R., Jones, C. I., \& Gibbins, J. M. (2013). Tangeretin regulates platelet function through inhibition of phosphoinositide 3-kinase and cyclic nucleotide signaling.Arteriosclerosis, Thrombosis and Vascular Biology, 33 (12), 2740-2749. doi:10.1161/ATVBAHA.113.301988.

Vaiyapuri, S., Roweth, H., Ali, M. S., Unsworth, A. J., Stainer, A. R., Flora, G. D., . . . Gibbins, J. M. (2015). Pharmacological actions of nobiletin in the modulation of platelet function. British Journal of Pharmacology, 172 (16), 4133-4145.

Varga-Szabo, D., Pleines, I., \& Nieswandt, B. (2008). Cell adhesion mechanisms in platelets.Arteriosclerosis, Thrombosis and Vascular Biology, 28 (3), 403-412. doi:10.1161/ATVBAHA.107.150474.

Vilahur, G., \& Badimon, L. (2013). Antiplatelet properties of natural products. Vascular Pharmacology, 59 (3), 67-75.

Wang, T. H., Bhatt, D. L., \& Topol, E. J. (2006). Aspirin and clopidogrel resistance: an emerging clinical entity. European Heart Journal, 27 (6), 647-654.

Yayeh, T., Im, E. J., Kwon, T.-H., Roh, S.-S., Kim, S., Kim, J. H., . . . Rhee, M. H. (2014). Hemeoxygenase 1 partly mediates the anti-inflammatory effect of dieckol in lipopolysaccharide stimulated murine macrophages. International Immunopharmacology, 22 (1), 51-58.

Figure legends

Fig. 1. Dieckol inhibits agonist-induced platelet aggregation . Washed platelets (A\&D from rat; $\mathbf{B} \& \mathbf{C f r o m}$ human) were pretreated with either dieckol or vehicle for $1 \mathrm{~min}$ in the presence of $1-\mathrm{mM} \mathrm{CaCl}_{2}$ and then stimulated with collagen, ADP, or thrombin for 5 min. (C) Human PRP was incubated with vehicle or various concentrations of dieckol in the presence of $10-\mathrm{mM} \mathrm{CaCl}_{2}$ for 1 min and then stimulated with ADP $(25 \mu \mathrm{M})$ for 5 min. (D) Scanning electron microscopy was performed to assess the extent of platelet aggregation and the ultrastructure. Representative scanning electron microscopy images $(5000 \times)$ of platelets treated with dieckol or vehicle [(a) resting, (b) vehicle, (c) dieckol $(2.5 \mu \mathrm{M})$, (d) dieckol $(5 \mu \mathrm{M})$, and (e) dieckol $(10 \mu \mathrm{M})]$ and then stimulated with ADP (b-e). (E) Chemical structure of dieckol. The graphs show the mean $\pm \mathrm{SD}$ of at least five independent experiments in duplicate. ${ }^{* *} p<0.01$ and ${ }^{* * *} p<0.001$ vs. control. WP, washed platelets.

Fig. 2. Dieckol inhibits ADP-stimulated $\left[\mathrm{Ca}^{2+}\right]_{i}$ elevation, ATP release, and serotonin secretion. (A) Washed platelets were loaded with a calcium fluorophore $(5 \mu \mathrm{M}$, fura-2/AM) for $1 \mathrm{~h}$. Fura 2/AM-loaded platelets were pre-treated with dieckol for 2 min at $37^{\circ} \mathrm{C}$ and stimulated with ADP $(10 \mu \mathrm{M})$. (B) Washed platelets were pre-treated with various concentrations of dieckol or vehicle for $1 \mathrm{~min}$ in the presence of 1-mM $\mathrm{CaCl}_{2}$ and stimulated with ADP $(10 \mu \mathrm{M})$ for $5 \mathrm{~min}$. (C-D) Human PRP was pre-treated with various concentrations of dieckol or vehicle for $1 \mathrm{~min}$ in the presence of $10-\mathrm{mM} \mathrm{CaCl}_{2}$ and then stimulated with ADP $(25 \mu \mathrm{M})$ for $5 \mathrm{~min}$. The concentration of ATP was assessed in a luminometer using an ATP assay kit and serotonin secretion was assessed using an EIA kit. Results represent the mean \pm SD of experiments performed on four independent days. ${ }^{*} p<0.05,{ }^{* *} p<0.01$, and ${ }^{* * *} p<0.001$ vs. control.

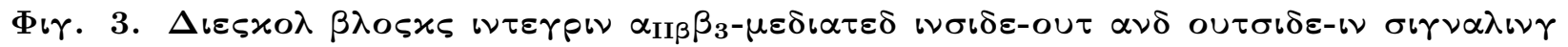

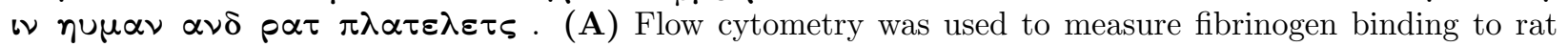
platelets after treatment with (a) resting, (b) vehicle, (c) dieckol $(2.5 \mu \mathrm{M})$, (d) dieckol $(5 \mu \mathrm{M})$, (e) dieckol (10 $\mu \mathrm{M})$, and (f) EGTA $(10 \mu \mathrm{M})$, and then platelet suspension was stimulated with ADP (b-f). (B) Bar graph summarizing the inhibitory effect of dieckol on fibrinogen binding to integrin $\alpha_{\mathrm{IIb}} \beta_{3}$. (C) Washed rat platelets 
were pre-incubated with different concentrations of dieckol, vehicle, or GR155053 (an $\alpha_{\text {IIb }} \beta_{3}$ inhibitor) and then loaded onto a fibronectin-coated plate and incubated for $90 \mathrm{~min}$ at $37^{\circ} \mathrm{C}$. Then, the assay was performed as described in the methods section. Bar graphs summarizing the inhibitory effect of dieckol on fibronectin adhesion. (D) Washed rat platelets were pre-incubated with vehicle (a), dieckol (b), or GR155053 (c) and then loaded onto a fibrinogen-coated cover glass and incubated for $60 \mathrm{~min}$ at $37^{\circ} \mathrm{C}$, washed and stained with SYTO-9, and scanned under confocal microscope. (E-G) Effect of dieckol on clot retraction in rat PRP was analyzed in vitro. Thrombin $(1 \mathrm{U} / \mathrm{mL})$ was added to initiate fibrin clot formation, and clot retraction was observed for $2 \mathrm{~h}$ at room temperature. (E) A representative image of clot retraction at $90 \mathrm{~min}$ in the presence or absence of dieckol or Y-27632 (Rho kinase inhibitor) and, (G) Kinetics of clot retraction were measured by Image-J software and clot surface areas were plotted as a percentage of retraction. (H) Human PRP was pre-incubated with vehicle (a), dieckol (b) or Y-27632 (c). Thrombin (1 U/mL) was added to initiate fibrin clot formation, and clot retraction was observed for 90 min at room temperature. (B, C, F, G) Results represent the mean $\pm \mathrm{SD}$ of experiments performed on four independent days. ${ }^{*} p<0.05,{ }^{* *} p<0.01$, and ${ }^{* * *} p<0.001$ vs. control.

Fig. 4. Dieckol inhibited ADP-stimulated MAPK activation and attenuated phosphorylation of PI3K/Akt in the $\mathbf{P}_{\mathbf{2}} \mathbf{Y}_{\mathbf{1 2}}$ signaling pathway. Washed platelets were pre-treated with different concentrations of dieckol and stimulated with ADP. Following termination of platelet aggregation, proteins were extracted from the platelets and analyzed for phosphorylation of respective molecules by immunoblotting. Immunoblot images are representative of the mean $\pm \mathrm{SD}$ of experiments performed on four independent days. ${ }^{*} p<0.05,{ }^{* *} p<0.01$, and ${ }^{* * *} p<0.001$ vs. control.

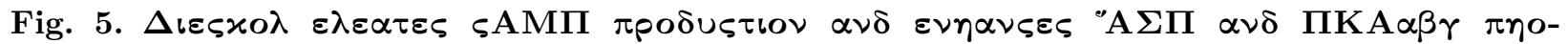
$\sigma \pi \eta \circ \rho \psi \lambda \alpha \tau \iota \mathrm{\nu}$. Washed rat platelets were pretreated with various concentrations of dieckol, vehicle, forskolin or SNP for $1 \mathrm{~min}$ in the presence of $1-\mathrm{mM} \mathrm{CaCl}_{2}$ and were then stimulated with ADP for 5 min. (A-B) After terminating the aggregation, the cAMP and cGMP immunoassays were performed using the cAMP and cGMP EIA kits, according to the manufacturer's protocol.(C) Proteins were extracted from the platelets and analyzed for phosphorylation of total VASP, p-VASPser157, and PKA $\alpha \beta \gamma$ by immunoblotting. Shown are representative immunoblot images and graphs of the means \pm SD and the data of at least four independent experiments. ${ }^{*} p<0.05$ and ${ }^{* * *} p<0.001$ vs. control. R, resting; C, control; FSK, forskolin; SNP, sodium nitroprusside.

Fig. 6. Dieckol inhibits pulmonary thromboembolism in mice without affecting hemostasis. (A-C) Mice were pre-treated with saline, dieckol (30 mg/kg i.p.), or ASA (100 mg/kg i.p.) once a day for 3 days $(\mathrm{n}=10$ in each group). One hour after the final administration, mice were challenged with a collagen + epinephrine mixture and checked for survival at $10 \mathrm{~min}(\mathbf{B}) .{ }^{* * *} p<0.001$ compared to the agonist-treated group. Arrows show the microvasculature occluded due to collagen-epinephrine induced thrombosis. (C) Graph shows occluded lung microvasculature. At least ten fields were examined, and the number of occluded vessels was counted in each group. (D)Mice were administered dieckol (30 mg/kg), aspirin (a positive control, $50 \mathrm{mg} / \mathrm{kg}$ ), or saline ( $\mathrm{n}=5$ in each group). Tail bleeding assay was performed as described in method section. The graph shows mean $\pm \mathrm{SD}$ of at least five independent experiments performed. ${ }^{* * *} p<0.01$ vs. control.

Fig. 7. Summary of inhibitory effects of dieckol on platelet intracellular signaling pathways.

\section{Hosted file}

Figures.pptx available at https://authorea.com/users/334567/articles/460534-pharmacologicalactions-of-dieckol-on-the-modulation-of-platelet-functions-and-thrombus-formation-viaintegrin-\%CE\%B1iib\%CE\%B23-and-camp-signaling 